\title{
Violence et politique : quatre éléments de réflexion à propos de la Colombie
}

Daniel Pécaut

\section{(2) OpenEdition \\ 1 Journals}

\section{Édition électronique}

URL : http://journals.openedition.org/conflits/197

DOI : 10.4000/conflits. 197

ISSN : $1777-5345$

Éditeur :

CCLS - Centre d'études sur les conflits lilberté et sécurité, L'Harmattan

\section{Édition imprimée}

Date de publication : 15 mai 1994

ISSN : 1157-996X

\section{Référence électronique}

Daniel Pécaut, «Violence et politique : quatre éléments de réflexion à propos de la Colombie », Cultures \& Conflits [En ligne], 13-14 I printemps-été 1994, mis en ligne le 14 mars 2006, consulté le 30 mars 2021. URL : http://journals.openedition.org/conflits/197 ; DOI : https://doi.org/10.4000/conflits. 197

Ce document a été généré automatiquement le 30 mars 2021.

Creative Commons License 


\title{
Violence et politique : quatre éléments de réflexion à propos de la Colombie
}

\author{
Daniel Pécaut
}

1 Cornelius Castoriadis ou Claude Lefort nous ont invité, il y a déjà quelque temps, à repenser "le politique", en cessant de le considérer comme une instance spécifique et "objective pour y repérer la manière dont les rapports sociaux se trouvent d'emblée pris dans un processus de "mise en forme" ou de "mise en scène". La position du Pouvoir, en extériorité par rapport au social, serait surtout celle d'un foyer de visibilité par lequel une société multiple parvient à percevoir son unité. Dans le cas de la démocratie, à suivre C. Lefort, le pouvoir ne serait, on le sait, qu'un lieu vide.

2 Une telle analyse, qui accorde une place généreuse à un imaginaire considère cependant, semble-t-il, comme allant de soi que cet imaginaire est un, comme la société qui s'y déchiffre, par delà les divisions qui la travaillent. C. Lefort dans ses contributions sur l'histoire politique du XIXe siècle, souligne la persistance d'un clivage radical entre diverses conceptions de l'ordre social, donc entre plusieurs imaginaires. Par ailleurs même si un imaginaire domine, des éléments étrangers peuvent le menacer : "les ennemis de la Révolution française" ou l'irruption possible de la barbarie tant crainte par Guizot.

3 Ces réflexions liminaires prennent davantage de relief quand on tente de réfléchir sur la Colombie où en dépit de l'existence de formes démocratiques relativement stables depuis des décennies, le politique ne paraît guère donner lieu à la production d'un "imaginaire" démocratique. De plus, à l'heure actuelle le pays est à nouveau marqué par des phénomènes de violence généralisée qui semblent enlever tout sens à l'idée d'"institution du social", ou du moins la compliquer singulièrement.

4 Ce n'est pas le lieu d'aborder ici le problème des représentations collectives qui accompagnent dans l'histoire colombienne la mise en oeuvre de procédures plus ou moins démocratiques. Cela nous amènerait à considérer le problème de la compatibilité entre des allégeances personnalisées, que certains disent imprégnées d'Ancien Régime, 
et le langage de la souveraineté populaire. Cela nous entrânerait aussi à nous interroger sur la compatibilité entre l'expérience d'une société "naturellement" divisée entre deux sous-cultures "politiques" transmises de génération en génération par l'intermédiaire des familles et des lieux, et la possibilité de penser un univers commun du politique: comment le Pouvoir pourrait-il acquérir fonction d'instauration symbolique alors que la société paraitt "originellement" clivée ? Disons-le une fois pour toute: la Colombie est l'un des seuls pays d'Amérique Latine à n'avoir pas connu les moments fondateurs suscités par les mobilisations populistes ou nationalistes. En l'absence d'une phase où "le peuple" a été réuni et où l'image de l'unité nationale a acquis quelque consistance, il n'y a pas lieu de s'étonner que l'Etat ait eu un rôle de régulation des relations sociales réduit, que la scène politique ait été constamment fragmentée, et que la "loi", loin d'avoir signification instituante, ait été perçue purement et simplement comme le produit de transactions fluides et précaires autorisant en réalité tous les accommodements et toutes les transgressions. La stabilité du régime pendant les seize années - de 1958 à 1974 - où les deux partis traditionnels se sont répartis par moitié tous les postes politiques et administratifs est vécue comme un arrangement ad hoc qui n'a rien à voir avec "l'invention démocratique" et ne peut susciter durablement beaucoup de ferveur. Si de semblables arrangements sont périodiquement conclus au long de l'histoire colombienne c'est qu'ils sont fort utiles, faute de mieux, pour faire barrage à la violence qui est perçue (ou imaginée) comme traversant de façon habituelle les rapports sociaux.

En parlant de "violence généralisée", nous voulons suggérer d'entrée de jeu que, lors de sa première explosion au cours des années 1946-1957, comme lors de sa deuxième explosion à partir de 1980, elle ne se laisse ramener ni à une guerre politique, ni à un conflit social. Si la violence prend appui sur des modalités de l'une et de l'autre, c'est pour les déborder. Du reste, elle engendre tôt ou tard leur décomposition. L'usage constant du terme "Violence" par les Colombiens laisse entendre qu'il s'agit dans leur esprit d'une force anonyme et incontrôlable qui s'affranchit des déterminations sociales pour être prise en charge en quelque sorte aléatoirement par les entités sociales et les individus les plus divers.

$6 \quad$ Il est évident pour tous qu'il est possible de repérer au départ les acteurs qui font de la violence une stratégie. Mais tout se passe comme si, au fur et à mesure que ces stratégies s'étendaient, elles suscitaient une autre violence qui faisait basculer tous les repères antérieurs, ceux ayant trait aux identifications politiques, mais aussi aux interactions sociales les plus ordinaires. La violence en se prolongeant, semblait devenir à son tour une forme normale et ordinaire de ces interactions. Les analystes qui se livrent au décompte et à la classification des victimes de "la violence" actuelle - le taux global d'homicides plaçant la Colombie dans les tous premiers rangs mondiaux -, ne manquent pas de souligner que seul un faible pourcentage peut être directement imputé à des causes politiques ou à l'action des groupes organisés de narcotrafiquants, les plus fort pourcentages étant liés aux règlements de compte ou aux disputes routinières. On a là, une relation circulaire: les stratégies organisées de violence ouvrant la voie à l'usage de la violence dans les litiges les plus banals. Cette diffusion de la violence favorise en retour le fait que les stratégies organisées de violence finissent par paraître dans l'ordre des choses. En étudiant les phénomènes récents de violence en Colombie, notre propos est bien d'analyser une situation où les turbulences de la société ne sont aucunement les prémisses d'une redéfinition de l'imaginaire politique: l'imaginaire politique y tient une place qui se réduit au fil du temps. Les diverses 
actions qui concourent à la violence relèvent bien d'un prosaïsme sans limites. Qu'elles s'inspirent au commencement de grandes eschatologies politiques, qu'elles prennent parfois et dans certaines zones - les traits d'une sorte de consentement de la population à un pacte hobbesien, qu'elles renvoient - parfois et un peu partout - à des jeux stratégiques entre les nombreux réseaux sociaux et économiques qui quadrillent le territoire, que parfois elles se réduisent, comme on vient de le dire, à des disputes interindividuelles, les actions violentes finissent par brouiller radicalement, non seulement les repères, mais les frontières mêmes du politique et, en définitive à entraver cette mise en visibilité qui est à la base du politique en même temps que le sentiment d'appartenance à une société. Ces quatre thèmes que nous esquissons ciaprès ne prétendent à aucune systématicité. Ce sont plutôt des esquisses juxtaposées de raisonnements destinés à alimenter le débat.

7 La division ami-ennemi et sa dissémination Dans la mesure où le recours à la violence est initialement le produit de la décision d'acteurs qui se veulent politiques, il passe par leur capacité à imposer une représentation de la politique dans les termes d'une division ami-ennemi. Dans le cas de la Violence des années 1946-1960, une telle représentation n'était guère difficile à mettre en avant. La conviction que la société était vouée à être éternellement partagée entre deux sous-cultures, porteuses de deux conceptions opposées de l'ordre social, instaurait le clivage ami-ennemi en fondement du politique. Dans la période récente, il en a été différemment. La référence à un tel clivage ne pouvait plus être présentée comme allant de soi, c'est-à-dire inscrite "naturellement" dans le social. Elle ne pouvait qu'être construite. D'où l'entreprise explicite des acteurs révolutionnaires tentant de démontrer que la violence était fabriquée par le régime lui-même et par les rapports sociaux dont il était l'expression, pour faire de leur propre violence le seul instrument susceptible de répondre à une situation d'injustice essentielle. En dehors d'un bref moment, vers 1980, cette lecture politique n'a pas réussi à s'imposer. La référence à la division ami-ennemi, fondatrice du rapport politique global, a été transposée, à mesure que les phénomènes de violence se diversifiaient en une manière de caractériser les types de litiges les plus divers. Soumise à une dissémination sans fin, elle a perdu son pouvoir de désigner le trait majeur de la société, et donc de maintenir sa teneur politique.

Figures du pacte hobbesien Comment rendre compte de l'encadrement par des réseaux de pouvoir, - guérillas, milices locales, organisations d'autodéfense ou paramilitaires, d'immenses secteurs de la population? Il nous semble que les conditions et les formes de "l'adhésion" des populations renvoient dans une large mesure à un processus préalable de carence ou de crise des modes globaux ou partiels de régulation sociale : la précarité des formes d'existence collective ou, dans certains cas, la fréquence des situations de "désaffiliation" selon le terme de R. Castel, en sont l'expression. Les zones où cette "adhésion" se produit le plus massivement sont, cela est abondamment démontré, celles qui ont été occupées récemment à la suite de migrations de provenances multiples. De surcroît l'emprise de l'Etat ne s'exerce pratiquement pas dans ces régions. Ce phénomène d'occupation de terres nouvelles a pris une ampleur accrue depuis vingt ans, notamment avec l'essor de l'économie de la drogue qui a attiré des ruraux traditionnels, mais également des urbains vers des régions périphériques ; mais il caractérise la Colombie depuis le XVIIIe siècle. Les paysans ou les "rebelles" (vaincus des guerres civiles, esclaves en cavale, ou travailleurs ruraux désireux d'échapper à la tutelle des propriétaires) y ont toujours vu le moyen de se soustraire, à l'influence des dominants. Les foyers ainsi créés loin des autorités ont été constamment 
perçus par le centre, comme "sans foi ni loi"; de fait, en l'absence de toute médiation institutionnelle, le traitement des litiges ne pouvait qu'y être expéditif. Cette situation s'est reproduite à une autre échelle avec la circulation soudaine d'abondantes quantités monétaires dues aux activités de la drogue. Même là où existaient auparavant des formes de coopération spontanée entre les nouveaux habitants, celles-ci se sont disloquées lorsque l'argent s'est répandu à profusion. La dépense ostentatoire et festive s'est imposée, accompagnée d'une dépense sous forme de violence.

Dans les zones de cultures commerciales plus classiques, les tensions sociales, si intenses fussent-elles, n'ont pu que rarement engendrer des actions collectives. La faiblesse des régulations institutionnelles, les stratégies politiques clientélaires qui alimentaient la fragmentation du territoire, le recours à la force privée comme procédé normal de solution des conflits, ne favorisaient guère de telles actions. Là où néanmoins les conflits arrivaient à se structurer par le biais de syndicats ou d'associations paysannes, l'implantation de protagonistes armés a suscité un mélange de violence "stratégique", impliquant la subordination des organisations sociales, et de violence ordinaire, minant la vie collective, ce qui, au bout de quelque temps, l'a réduite à néant.

Les identités collectives de type culturel n'ont, par ailleurs, jamais été très répandues dans le cas colombien. Le métissage très ancien, les incessants déplacements de population, et surtout l'omniprésence des réseaux liés aux deux partis traditionnels qui ne répondaient à aucune (ou si peu) géographie culturelle ou opposition sociale, sont autant de facteurs qui peuvent l'expliquer. L'appartenance aux partis traditionnels et à l'Eglise catholique, garante jusqu'à 1970 de l'ordre social, ont en fait longtemps tenu lieu pour la majorité d'instruments de définition culturelle.

11 Ces éléments, de longue durée ou de conjoncture, permettent de comprendre la fragilisation des liens collectifs, mais ils ne suffiraient pas à interpréter "l'adhésion" aux nouveaux réseaux armés si, de surcroît, la violence très concrète n'avait touché d'amples secteurs de la population. Au moins dans les zones de colonisation, la référence à Hobbes paraît justifiée. Il se produit bien, face à des expressions multiples de violence, une manière de pacte pour remettre à un souverain local, en l'occurrence à la guérilla ou aux militaires et paramilitaires, le monopole de la force. Dans d'autres zones, "l'adhésion" peut emprunter d'autres voies. A la place de Hobbes, il faut peutêtre dans leur cas mettre tout simplement Olson : la contrainte est présentée comme la condition d'une "reconstruction" de la collectivité.

Décision ou imposition : il est en tout cas manifeste que la référence à "l'ordre" est bien essentielle. Ce n'est pas un hasard si les sujets du nouveau souverain justifient l'abandon de leur liberté par le fait qu'enfin il existe un souverain pour "rendre la justice". Que cette justice passe par de nombreuses mises à mort ne fait que renforcer le sentiment que les sujets souhaitent bloquer la diffusion horizontale de la violence en en réservant l'exercice à "l'Un" au dessus d'eux.

13 A la différence de Hobbes le souverain peut facilement être remplacé. Les paramilitaires peuvent prendre la place de la guérilla à condition qu'ils instaurent "l'ordre" à leur tour. Une seconde fois l'on revient en quelque façon à Olson : dans une situation d'extrême violence et d'extrême atomisation des sujets (produit de cette violence), le seul critère (ou presque) d'orientation des conduites est utilitariste: il commande de faire le nécessaire pour préserver ses intérêts (à commencer par la conservation de la vie) en s'inclinant devant le souverain de service, et à se taire pour le 
reste, même si on n'en pense pas moins. C'est si l'on veut, l'une des marques du "prosaïsme" des phénomènes de violence.

Interactions stratégiques et dégradation du contenu politique de la violence Quand les phénomènes de violence se généralisent, les interventions de tous les protagonistes organisés peuvent s'analyser en termes stratégiques, comme un jeu fluide entre des réseaux disposant de diverses ressources de pouvoir. Mais cela implique que, même dans le cas des protagonistes qui se présentent comme porteurs de projets sociaux ou politiques, un effacement progressif de ces projets se produit. Nous voulons ainsi suggérer qu'au moment où les interactions des protagonistes organisés entraînent une redéfinition du champ social comme ensemble complexe et concurrentiel de réseaux d'action et de régulations locales et partielles, la référence au politique tend à s'estomper.

15 Le constat de la "dépolitisation" des phénomènes de violence qui s'étendent n'a rien d'original. Il est accepté par la plupart des analystes et expliqué par deux facteurs. L'un est la tendance des protagonistes politiques: guérillas mais aussi les militaires ou paramilitaires, à mettre en oeuvre des pratiques, comme le prélèvement systématique de rançons après enlèvement, ou la taxation de la population, qui finissant par apparaître comme des fins par elles-mêmes, semblent relever du banditisme. L'autre renvoie aux interférences entre protagonistes politiques et protagonistes "économiques" (narcotrafiquants).

16 Ces deux facteurs ne peuvent être négligés. Mais la "dépolitisation" peut, nous semble$\mathrm{t}$-il, être mieux comprise si on considère tout simplement que les acteurs initialement politiques sont pris dans des logiques d'interaction stratégique où ils se redéfinissent par rapport aux ressources qu'ils contrôlent, aux régulations qu'ils soutiennent, aux échanges et conflits qui les lient aux autres acteurs, ce positionnement gommant peu à peu leur dimension proprement politiques.

17 Dans un tel système d'action, les guérillas, par exemple, prennent l'aspect de simples réseaux de pouvoir disposant de moyens de coercition. Leur montée en puissance dès le début des années 1980 ne peut être dissociée du profit qu'elles ont tiré de leur contrôle sur les zones de production de drogue et, peu à peu, sur les zones de production d'autres matières premières: pétrole, or, émeraudes, produits agricoles, voire sur certaines zones d'implantation industrielle. Leur expansion territoriale, apparemment impressionnante puisqu'elles font désormais acte de présence sur près de la moitié des municipes du pays, a largement obéi au souci d'élargir leur contrôle sur les richesses nationales. Depuis quelque temps, elles s'efforcent, avec un succès indéniable, de l'étendre aussi sur la gestion financière des municipes. Cette stratégie passe par la consolidation de leur emprise sur les territoires qu'elles considèrent comme stratégiques. Elle conduit également à une transformation des rapports avec les populations de ces territoires. Les querelles ne prétendent plus depuis longtemps "mobiliser" ou endoctriner les populations. Elles cherchent seulement à assurer leur emprise sur elles, y compris en s'efforçant d'exercer un monopole sur les conditions d'accès aux emplois locaux.

Il est donc légitime de les considérer comme de simples réseaux de pouvoir, interférant avec d'autres réseaux de pouvoir (narcotrafiquants, militaires, entrepreneurs) qui possèdent également une certaine capacité de contrôle sur les ressources et les populations. Les moyens de violence ne sont jamais qu'une ressource supplémentaire. Les jeux stratégiques entre ces divers réseaux se traduisent en "régulations croisées", 
pour reprendre une expression de la sociologie des organisations, qui, en fonction de leurs caractéristiques, définissent les nouvelles scènes territoriales. La preuve est que de nouvelles régions se sont constituées, comme résultat, des interactions violentes entre réseaux.

Jusqu'à un certain point, on peut admettre, conformément à ce que proposent certains des tenants de l'analyse stratégique ${ }^{1}$, que l'action de l'Etat (à travers les administrations civiles ou les militaires) s'insère elle-aussi, au moins, au plan local, dans les systèmes d'action ainsi constitués en répondant à des stratégies diversifiées et entretenant divers rapports avec les actions des autres protagonistes. Ainsi parvient-on à généraliser le raisonnement stratégique en éclairant les jeux complexes entre réseaux.

Reste une question à la fois théorique et pratique, fondamentale : dans quelle mesure ce raisonnement stratégique ne dissout-il pas la dimension proprement politique? Les stratégies des protagonistes antérieurement "politiques" de la violence peuvent être mises en lumière par cette conceptualisation. Elles permettent de décrire des concurrences, des transactions, des alliances, des accords, des confrontations, des régulations fluides, et tout cela est, en l'occurrence, fort utile. Mais le politique ne se résume pas à ces jeux d'influences, plus que l'action collective à une "mobilisation de ressources"; il renvoie à un autre plan, celui des décisions, au sens fort, celui qui concerne le non-négociable, celui des institutions, celui de la légitimation conçue comme autre chose que le produit des transactions ${ }^{2}$.

21 Dès lors, la conversion des acteurs colombiens en réseaux immergés dans des systèmes fluides et locaux d'interactions doit être déchiffrée en fonction de ce qu'elle indique : la perte des références politiques qui se manifeste à la fois par la manière dont les conflits sociaux sont oblitérés et par la manière dont les justifications politiques perdent toute crédibilité.

L'impossibilité d'un récit d'ensemble de la violence On ne peut qu'être surpris de noter que, plus de trente ans après son "achèvement", le premier épisode de violence se dérobe toujours à l'opération de "mise en intrigue". Comme si il s'agissait d'une trame distendue laissant partout subsister des vides. Ceux qui tentent d'évoquer cette période, qu'ils l'aient ou non vécue, oscillent généralement entre trois positions. La première consiste à reprendre à son compte l'identification à l'un des camps politiques en conflit, et donc à en reproduire les argumentations. La seconde est de citer quelques événements, presque toujours les mêmes, comme s'ils tenaient lieu de récit par euxmêmes. La dernière est de faire référence à l'expérience personnelle construite, elle aussi, comme une addition d'événements fortuits. La multiplicité des acteurs, des scènes, des logiques contribuent à ruiner l'élaboration d'un récit d'ensemble. A quoi s'ajoute le fait que le dénouement très prosaïque donné à cet épisode, sous l'aspect d'une répartition du pouvoir entre les deux partis précédemment ennemis, revient à couvrir d'un voile d'ignorance ce qui s'est produit et à interdire toute interrogation sur son sens et son injustice. Que cette catastrophe ait été ainsi laissée à l'état brut n'est du reste pas sans rapport avec l'attrait exercé par les perspectives de la violence proprement révolutionnaire sur les descendants de familles touchées par la violence : ils ne font sans doute que chercher à donner sens à l'inexplicable du passé.

23 Sans doute est-ce là une description qui vaut pour d'autres situations de "violence généralisée". Elle vaut en tout cas pour les phénomènes récents de violence. Là encore existe la conviction partagée qu'il est impossible de parvenir, à leur propos, à une mise 
en intrigue globale. Des entretiens réalisés dans les régions de violence intense, l'on peut dégager trois indications :

a) Les récits individuels, liés aux expériences de "la violence", ne s'insèrent pas dans un récit plus large ;

b) les seuls récits d'ensemble de la violence ont l'aspect de mythes, et sont largement soustraits à l'histoire ; c) les "faits" ont statut d'un réel qui ne se laisse pas aisément insérer dans une histoire signifiante.

a) - Dans les récits issus des expériences individuelles, les références à "la violence" sont partout présentes. La trajectoire de vie est souvent décrite comme celle d'un déplacement spatial ininterrompu, la "violence" étant au départ, intervenant au cours de chaque réorientation de la trajectoire, se retrouvant à l'arrivée, arrivée qui n'est d'ailleurs jamais qu'une nouvelle étape. En arrière-fond se trouve toujours la violence des années 1940-50. Mais les faits cités au titre de "faits de violence" récents comprennent aussi bien des incidents, "politiques" ou non, que des accidents matériels ou naturels (une inondation qui détruit les cultures). Cela suggère que ces faits ne sont pas toujours imputables à des êtres humains et que, même quand ils le sont, on les met souvent au compte d'une "force inhumaine" cachée derrière les êtres humains, ce qui est une manière de désigner l'excès ou le non-représentable. Mais il semble surtout aller de soi qu'il n'est d'autre manière de faire référence à la violence qu'en racontant sa propre histoire, ce qui revient à admettre que "la violence" ne peut se dire qu'à travers une infinité $\mathrm{d}$ 'histoires qui ne constituent aucun ensemble.

b) - Sans doute la Violence d'ensemble est-elle aussi évoquée. Elle l'est au titre d'une trame qui est celle de toute l'histoire colombienne, mais paradoxalement cette trame est présentée comme elle-même non-historique: c'est la même violence qui est supposée être continuellement à l'oeuvre au XIXe et au XXe siècle (alors même que l'on sait que les années 1910-1945 ont été exemptes ou presque de violence). Quant aux épisodes de violence aiguë, ils paraissent régis par le principe de répétition : peu nombreux sont ceux qui doutent que la violence actuelle soit la reprise de celle des années 1945-1960, et que cette dernière soit la reprise de la guerre civile. L'oeuvre de Garcia Marquez rend évidemment compte, mieux que toute oeuvre sociologique, des structures mythiques qui supportent les conceptions de la violence.

c) - Les "faits" résistent à leur insertion dans un récit qui ne soit pas celui de l'expérience individuelle. Ce qui n'est pas vraiment étonnant. Si le mythe est la seule représentation possible de la violence, et si l'histoire d'ensemble est impossible, les faits sont là pour faire jonction, sous forme de réel indépassable, entre le mythe et l'expérience, et tiennent finalement lieu d'histoire. Pour le reste, ces faits bruts se prêtent à toutes les fantaisies interprétatives. La généralisation de la violence s'accompagne en effet de la dissolution d'une opinion publique susceptible de décider de leur portée et de leur "origine". On a pu s'en apercevoir lors des séries d'assassinats spectaculaires perpétrés en 1989-1990. Alors que l'on pouvait de l'extérieur les imputer avec quelque vraisemblance à des secteurs définis (les narcotrafiquants par exemple), ils faisaient l'objet des rumeurs qui en rendaient responsables les forces les plus fantastiques. 


\section{NOTES}

1. Par exemple, Friedberg (E.), Le Pouvoir et la Règle, Paris, Seuil 1993.

2. Comme le soulignent J. Leca et B. Jobert dans leur commentaire critique de l'Acteur et le système, Cf. "Le dépérissement de l'Etat" : à propos de l'Acteur et le système de M. Crozier et E. Friedberg, Revue française de science politique, 1980, p. 1125-1170.

INDEX

Mots-clés : violence, forces paramilitaires

Index géographique : Amérique du Sud, Colombie

\section{AUTEUR}

DANIEL PÉCAUT

Ce texte a été rédigé dans le cadre d'une journée d'études du CEMS. 